\title{
Metagenomic and metabolic shift on morbid obese patients undergoing bariatric surgery
}

\author{
S. Román-Sagüilloº , M.V. García-Mediavilla ${ }^{1,2}$, M. Juárez-Fernández ${ }^{1}$, D. Porras ${ }^{1}$, P. Linares ${ }^{3}$, \\ M. Ballesteros-Pomar ${ }^{4}$, A. Urioste-Fondo ${ }^{4}$, B. Álvarez-Cuenllas ${ }^{3}$, J. González-Gallego ${ }^{1,2}$, \\ S. Sánchez-Campos ${ }^{1,2}$, F. Jorquera ${ }^{3}$ and E. Nistal ${ }^{1,2}$ \\ ${ }^{1}$ Instituto Universitario de Biomedicina (IBIOMED), Universidad de León, León, Spain, \\ ${ }^{2}$ Centro de Investigación Biomédica en Red de Enfermedades Hepáticas y Digestivas (CIBERehd), Instituto de Salud \\ Carlos III, Madrid, Spain, \\ ${ }^{3}$ Complejo Asistencial Universitario de León, Servicio de Aparato Digestivo, León, Spain and \\ ${ }^{4}$ Complejo Asistencial Universitario de León, Departamento de Endocrinología y Nutrición, León, Spain
}

Obesity has emerged as one of the major global public health concerns, associated with a significant morbidity and mortality. Obesity clinical management demands increasing efforts for new therapeutic targets and innovative approaches. The link between gut microbiota and obesity has been thoroughly demonstrated, pointing gut microbiota manipulation as a promising tool to address this issue $^{(1)}$. Bariatric surgery is a reliable resource to deal with severe cases when other approaches have failed. However, it has been stated that it may have a profound impact on gut microbiota which is still poorly understood ${ }^{(2)}$. We aim to characterise the changes in faecal microbiome and metabolome associated with bariatric surgery on morbid obese patients.

Ten patients (20-60 years old) diagnosed with obesity not treated with antibiotics 1 month before the study were recruited by Endocrinology Service of Complejo Asistencial Universitario de León. Fecal samples were collected before and after bariatric surgery and processed for metabolomics and metagenomic analysis. Microbiota composition was identified by 16S rRNA Illumina MiSeq sequencing. Polar metabolites were quantified by liquid chromatography-mass spectrometry and gas chromatography-mass spectrometry was employed to determine short chain fatty acids (SCFAs) concentration. Kruskal-Wallis followed by Wilcoxon test for paired samples allowed identification of varying taxa. Univariant analysis according to fold change and significant Wilcoxon test, and multivariate analysis based on PLS-DA models were employed to estimate differences associated to surgery.

Bacterial communities were modified by bariatric surgery, tending to cluster separately on $\beta$-diversity plot based on UniFrac distance, while $\alpha$-diversity remained unaffected. More remarkable differences were detected at phylum and genus levels. Relative abundance of Tenericutes, Firmicutes, Saccharibacteria (phyla), Coprococcus, Lactococcus and Lachnospira (genera) decreased after surgery while increases in Butyricimonas, Parabacteroides and Slackia were detected. Fecal metabolome composition was deeply modified by bariatric surgery. All predominant SCFAs (acetate, propionate and butyrate) experimented a significant decrease upon surgery $(-52.2 \pm 15.40 \%,-46.2 \pm 11.92 \%$ and $-63.5 \pm 9.26 \%$, respectively). Moreover, metabolomic analysis highlighted the contribution of a subset of metabolites with a great performance on classifying samples in pre and post-operated. Among these compounds, intermediates of tricarboxylic acids cycle like citrate and malate, amino acids like aminolevulinate and histidine and others like taurine, glyoxylate or choline (all experimenting decreases) highlighted as the ones with larger differences following bariatric surgery, being considered hallmarks of the procedure and a potential fingerprint of weight loss in obese patients.

Bariatric surgery reshapes gut microbiota composition and produces significant alterations in the metabolome which can be related to the metabolic outcomes of the procedure. More studies are needed to understand the role of the microbial and metabolomic fingerprint of bariatric surgery in obesity and weight loss therapies.

\section{Acknowledgements}

Supported by BFU2017-87960-R and GRS1888/A/18. CIBERehd is funded by Instituto de Salud Carlos III.

\section{References}

1. Abenavoli L, Scarpellini E, Colica C et al. (2019) Nutrients 11, 2690.

2. Magouliotis D, Tasiopoulou V, Sioka E et al. (2017) Obes Surg 27, 1345-1357. 